\title{
Rule-specific dimensional interaction effects in concept learning
}

\author{
J. STEVEN REZNICK, R. DANIEL KETCHUM, and LYLE E. BOURNE, JR. \\ University of Colorado, Boulder, Colorado 80309
}

\begin{abstract}
Garner (1976) has identified two main types of dimensional interactions, integrality and separability, which are likely to affect concept learning. Evidence available to Garner suggested that integral stimuli facilitate concept learning, but these studies investigated only concepts based on the conjunctive or some simpler rule. The present experiment assessed the integralityseparability effect in concept learning over a variety of bidimensional rules. The results indicate that integral dimensions facilitate conjunctive rule learning but that separable dimensions facilitate the acquisition of conditional and biconditional rules. Thus, dimensional interaction effects are rule specific. In particular, any rule which requires a precise discrimination of the truth table class structure of the stimulus population will be facilitated when stimulus dimensions are separable.
\end{abstract}

A logical concept based on a binary operator or rule assigns positive (exemplar) or negative (nonexemplar) status to each of four possible stimulus classes: TT, both critical features present in the stimulus; TF, the first present and the second absent; FT, the first absent and the second present; and FF, both absent. For a variety of reasons, research has focused on four logical rules: the conjunctive rule, in which each example of the concept must contain both features; the disjunctive rule, in which examples of the concept have both or either feature; the conditional, in which, if a stimulus has the first feature, it must also have the second to be an example of the concept; and the biconditional, in which stimuli belonging to the concept have both or neither feature.

These rules are not of equal difficulty to learn or use. When subjects are told the relevant features and must acquire the rule defining the concept, conjunctive learning is more rapid than disjunctive, disjunctive more rapid than conditional, and conditional more rapid than biconditional (Bourne, 1970; Bourne \& Guy, 1968; Salatas \& Bourne, 1974). In some experiments two clusters emerge, with the conjunctive-disjunctive cluster learned more rapidly than the conditionalbiconditional cluster (Bourne, 1967; Bourne \& O’Banion, 1971; Reznick \& Richman, 1976).

Most literature to date has focused on the nature of the rule itself. The main thrust of this approach is, "What is it about the rule that makes it more or less difficult to learn?" To this end, theorists have proposed

The authors gratefully acknowledge the work of John E. Morrison and Charles L. Richman as an impetus for the present experiment. This research was supported by NSF Grant BNS7681416 and is Publication 70 from the Institute for the Study of Intellectual Behavior, Boulder, Colorado. Send reprint requests to J. Steven Reznick, Psychology Department, William James Hall, Harvard University, Cambridge, Massachusetts 02138. and explored numerous potential causes of differential rule difficulty, such as the number of positive instances to be learned, the relative size of the positive and negative categories, and the complexity of the logical operations (Bourne, 1974). Recently, our understanding of this problem has been improved by focusing on rule difficulty as an interaction between initial response tendencies of the person learning the rule and the rule to be learned. For example, Reznick and Richman (1976), Salatas and Bourne (1974), and Sawyer and Johnson (Note 1) have developed models in which rule difficulty results from differences between the response assignments a person expects and those that are required by the rule.

The present experiment investigates an additional factor that may be involved in the subject-rule interaction, namely, the perceptual characteristics of the stimuli which manifest the rule. Using a wide range of experimental paradigms, Garner (1974) has demonstrated that stimulus dimensions may interact perceptually in various ways. Of particular relevance to concept learning is the distinction between separable and integral dimensions. In a stimulus composed of features from two separable dimensions, the features are perceived as isolated, unrelated events. With integral dimensions, the features interact to produce a new, emergent relation.

The stimuli used in a concept problem usually vary on several dimensions, only a limited number of which are relevant to the concept. Of primary interest in the present research is the effect of dimensional interactions within the set of relevant dimensions. If the set of relevant dimensions is integral and they are perceived as going together, this could facilitate the process of separating relevant from irrelevant dimensions and, as a consequence, facilitate the determination of a rule that applies within the relevant set. On 
the other hand, logical rules require differential treatment of combinations of dimension values. Integral stimuli may mask the individuality of dimensions and interdimensional combinations, thus retarding concept learning.

After reviewing the available literature, Garner (1976) concluded that concept learning proceeds more rapidly with integral relevant dimensions. On the basis of these results, he reasoned that subjects might learn the response assignments of individual stimuli rather than the logically defined dimensional concepts in many concept learning situations. This possibility, of course, seriously questions the interpretation of a considerable body of concept learning research. It therefore seemed advisable to assess the generality of Garner's conclusions, which were based primarily on studies using conjunctive concepts. This study examines the effect of stimulus integrality in concept problems based on four different rules: conjunctive, disjunctive, conditional, and biconditional.

\section{METHOD}

\section{Subjects}

The subjects were 144 University of Colorado introductory psychology students who participated in the experiment as part of their course requirements. There was approximately equal representation by sex in each condition.

\section{Apparatus}

The stimuli were presented on slides. Each slide contained one value on each of three dimensions. The dimensions and their values were color (blue, red, or yellow), size (large, medium, or small), and shape (square, triangular, or circular). In integral stimuli all values were represented in a single figure centered in a slide, for example, a large blue square. In separated stimuli each dimension was represented separately. The upper left corner of the slide contained the black outline of a mediumsized square, triangle, or circle. The upper right corner contained the black outline of a large, medium, or small trapezoid. The bottom left corner contained a medium trapezoid in blue, red, or yellow.

A console in front of the subject contained two lamps-one labeled "correct" and one labeled "incorrect"- and two buttons-one labeled "+" and one labeled "-." Automated equipment recorded responses, determined and delivered feedback, and advanced the slide projector. After each response, the slide remained in view while the subject received a 3 -sec feedback signal. The slide projector then advanced immediately and the next trial began. The slide projector tray was recycled, if necessary, after each 32-trial run.

\section{Procedure}

Subjects were told that they would see a series of stimuli to which they should respond either positively or negatively. Each response would be correct or incorrect and their task was to give all correct responses. The domain of possible stimuli was described and demonstrated. Subjects were told that the rule for determining correct responses would be based on the absence or presence of two particular values on two different dimensions, and a card naming the values was left in the subject's view for the entire experiment. The experimenter explained how focusing on two values would yield four types of stimuli with both, just one, just the other, or neither attribute. To insure the subject's understanding of this distinction, he was asked to describe verbally the first four stimuli of the experiment in these terms. Subjects were allowed to respond at their own pace. After every 32 trials, the experimenter briefly reviewed the instructions. Criterion was 10 consecutive correct responses.

Each subject solved a problem based on one of four rules (conjunction, disjunction, conditional, or biconditional) with either integral or separated stimuli. Within each cell of the 2 by 4 design were counterbalanced three pairs of relevant attributes (large and circular, triangular and blue, or small and red) and two stimulus orders. One order began TT, TF, FT, FF and the other began TT, FF, TF, FT. In both orders, representation from truth-table classes was equated in each block of eight stimuli.

\section{RESULTS}

A probability level of .05 defined the rejection region for all statistical tests. Since analysis of both trials and errors to criterion yielded comparable results, only error to criterion data are reported. Table 1 contains the mean errors to criterion for each cell. A pre-ANOVA data check revealed a significant inequality of variance that could be rectified by a $\log _{10}(X+1)$ transformation. In a 4 (rules) by 2 (stimulus conditions) ANOVA on the transformed error scores, there was a significant rule effect $[\mathrm{F}(3,136)=41.37]$ and a significant Rule by Stimulus interaction $[\mathrm{F}(3,136)=2.67$, MSe $=.087]$. Duncan's new multiple range test indicated that a conjunctive-disjunctive rule cluster was easier than a conditional-biconditional cluster, with all between-cluster differences significant and all within-cluster differences not significant. A Duncan test on the Rule by Stimulus groups indicated that beyond the cluster differences, the only additional significant difference occurred between the conjunctiveintegral group and both the disjunctive-separated and conjunctive-separated groups, the former being easier.

To determine whether the stimulus manipulation differentially affected truth-table classes, trial to last error on a class was computed. Tests on these differences revealed that the TF class took fewer trials to learn with separated as compared to integral stimuli $[t(34)=2.65]$ for the conditional rule and that learning the FF class was facilited by separated stimuli in both the conditional $[\mathrm{t}(34)=4.10]$ and the biconditional $[\mathrm{t}(34)=2.26]$ rules.

\section{DISCUSSION}

These results suggest that the effect of stimulus dimensionality is rule specific rather than general. We found, as did

Table 1

Mean Errors to Criterion

\begin{tabular}{lrr}
\hline \multirow{2}{*}{ Rule } & \multicolumn{2}{c}{ Stimuli } \\
\cline { 2 - 3 } & Integral & Separated \\
\hline Conjunctive & 1.889 & 4.278 \\
Disjunctive & 3.056 & 3.389 \\
Conditional & 13.667 & 11.278 \\
Biconditional & 13.444 & 10.833 \\
\hline
\end{tabular}


the experiments reviewed by Garner, that unitary stimuli can be facilitative; the effect appears, however, only with the conjunctive rule. With the disjunctive rule, there is no apparent dimensionality effect; with the conditional and biconditional rules, separated stimuli facilitate acquisition. These data raise two main questions: First, how can this pattern of results be explained, and, second, can we integrate the results of this experiment with those reviewed by Garner.

Integral relevant attributes fuse into a unit. A person should see an integral stimulus as, for example, a blue square or not a blue square. If the attributes are separable, a person is more likely to see the dimensional structure with a TT stimulus as composed of both blue and square, a TF as blue and not square, etc.

In a conjunctive concept, TT stimuli are positive and all other truth-table classes are negative. Since integral stimuli are expected to generate this very distinction, integral stimuli should be facilitative for conjunctive concepts. For the other rules, a simple TT vs. not-TT approach does not solve the problem. In these cases, it is necessary to make other distinctions. In disjunction, the distinctions are again relatively simple, but different from the distinction inherent in integral stimuli. In a disjunctive concept, TT, TF, and FT are grouped together and are distinct from FF. As expected, integral stimuli promote no significant facilitation. Since dimensional structure is important in disjunction, we might have expected separable stimuli to facilitate acquisition; however, as Reznick and Richman (1976) observed, many people naturally take a disjunctive approach to all rules. For these people disjunction is learned without their necessarily learning the dimensional structure; hence, separable stimuli would have a less facilitative effect.

In conditional and biconditional concepts, an understanding of truth-table structure is crucial. In the biconditional, TT and FF responses totally differ from TF and FT responses and, in the conditional, they partially differ. Past research has shown that, for these rules, certain truth-table classes are particularly difficult to learn. In the conditional, TF and FF are hardest; in the biconditional, FF is hardest (Bourne \& Guy, 1968; Reznick \& Richman, 1976). For these rules, the data of this experiment show that separable stimuli, probably by heightening dimensional salience, facilitate acquisition of these difficult responses.

In retrospect, one aspect of our experimental procedure might have worked against finding a large improvement in complex rule acquisition with separated stimuli. We expected stimuli to facilitate the use of an approach whereby people coded the stimuli into a logical truth-table description. It should be noted that all subjects were informed about the nature of truth-table classes and actually practiced describing the stimuli in truth-table terms. We did this because our experience has been that complex rule-learning problems are quite difficult and that, without adequate instructions, subjects often fail to solve them. By giving training, we promote the tendency to code the stimuli in truth-table form in both stimulus conditions and therefore might have reduced the expected differences.

Regarding the second question, the present experiment is compatible with those reviewed by Garner. Bourne and Parker (1964) and Siegel (1969) found that unitary stimuli facilitated concept attainment, but both studies examined only conjunctive concepts. It is questionable whether Garner's other two pieces of evidence, Shepard, Hovland, and Jenkins (1961) and Wallach (1962), are comparable to the type of concept learning discussed here. In these experiments, unitary stimuli facilitated acquisition of a biconditional concept. But both employed a stimulus population varying on binary dimensions and consisting of only eight unique stimuli. Reznick and Richman (1976) investigated the effects of varying the number of alternative attributes on relevant dimensions. They suggested that, when the number of alternatives was reduced to two, people tended to learn responses to particular stimuli rather than generalized responses to truth-table classes of stimuli. Since the two experiments cited by Garner required that the subject learn responses to only eight different stimuli, it soems quite plausible that the subjects learned stimulus-response associations rather than a concept per se. Following Garner, if the concept is learned via individual stimulus, integral stimuli should be facilitative. If, as in the present experiment, problems are sufficiently complex to necessitate truth-table classification, then separated stimuli can facilitate their acquisition.

Stimulus sets with integral or separable dimensions are psychologically different. With integral stimuli the dimensions fuse into a unit and facilitate partitions that parallel this organization. With separable stimuli the dimensions maintain their discrete identities, and partitions that are built upon specific assignments for dimensional combinations are facilitated. Garner's original expectations are supported by the results of the present experiment. Rules that are defined by logical combinations of dimensional values are more easily acquired when the stimuli facilitate perceiving their dimensional structure. However, it must be appended that some rules may be learned without knowledge of their dimensional structure and may, in fact, be facilitated by stimuli that, in effect, mask that structure.

\section{REFERENCE NOTE}

1. Sawyer, C. R., \& Johnson, P. J. A concept learning model. Paper presented at the meeting of the Rocky Mountain Psychological Association, 1970.

\section{REFERENCES}

Bourne, L. E., JR. Learning and utilization of conceptual rules. In B. Kleinmuntz (Ed.), Memory and the structure of concepts. New York: Wiley, 1967.

Bourne, L. E., JR. Knowing and using concepts. Psychological Review, 1970, 77, 546-556.

Bourne, L. E., JR. An inference model for conceptual rule learning. In R. Solso (Ed.), Contemporary issues in cognitive psychology: The Loyola symposium. New York: Wiley, 1974.

Bourne, L. E., JR., \& GuY, D. E. Learning conceptual rules: I. Some interrule transfer effects. Journal of Experimental Psychology, 1968, 76, 423-429.

Bourne, L. E., JR., \& O'Banion, K. Conceptual rule learning and chronological age. Developmental Psychology, 1971, 5, 525-534.

Bourne, L. E., JR., \& PARker, B. K. Differences among modes for portraying stimulus information in concept identification. Psychonomic Science, 1964, 1, 209-210.

GARNER, W. R. The processing of information and structure. Potomac, Md: Lawrence Erlbaum, 1974.

GARNER, W. R. Interaction of stimulus dimensions in concept and choice processes. Cognitive Psychology, 1976, 8, 98-123.

Reznick, J. S., \& Richman, C. L. Effects of class complexity, class frequency, and preexperimental bias on rule learning. Journal of Experimental Psychology: Human Learning and Memory, 1976, 2, 774-782.

Salatas, H., \& Bourne, L. E., JR. Learning conceptual rules: III. Processes contributing to rule difficulty. Memory \& Cognition, 1974, 2, 549-553.

ShePard, R. N., Hovland, C. I., \& Jenkins, H. M. Learning and memorization of classifications. Psychological Monographs, 1961, 75(13, Whole No. 517).

SIEgEl, L. S. Concept attainment as a function of amount and form of information. Journal of Experimental Psychology, 1969, 81, 464-468.

Wallach, L. The complexity of concept-attainment. American Journal of Psychology, 1962, 75, 277-283.

(Received for publication June 19, 1978.) 